\title{
Medical Management of Unruptured Ectopic Pregnancy with Methotrexate and its Outcome
}

\author{
Dr.Sheila K.Pillai *1. Dr.Bhuvana Srinivasan ${ }^{2}$, Dr.Suganya Devi ${ }^{3}$ \\ 1. Associate Professor SRIHER Chennai \\ 2. Associate Professor SRIHER Chennai \\ 3. Post Graduate Student SRIHER Chennai \\ 1.drsheilasunil@gmail.com \\ 2.kritibhuvana@yahoo.co.in \\ 3.devi.arjunan@gmail.com
}

\begin{abstract}
Ectopic pregnancy (EP) is a classic gynecologic emergency and remains a major cause of maternal morbidity and mortality. The combination of serum beta hCG titre and transvaginal sonography have supplanted physical examination findings as the most accurate diagnostic tool for determining the site of ea rly gestation.

Early diagnosis by transvaginal scan has facilitated the move towards medical therapy with methotrexate for most women with unruptured ectopic pregnancies. Our study aims to analyze the outcomes of medically managed unruptured ectopic pregnancies treated with methotrexate and to correlate them with serum $\beta$-hCG levels. Patients who fulfilled the inclusion criteria for medical management of unruptured ectopic pregnancy $(\beta-h C G>1500 \mathrm{mIU} / \mathrm{mL}$ and $<10000 \mathrm{mIU} / \mathrm{mL}$ ) with transvaginal scan feature s suggestive of unruptured ectopic pregnancy were included in the study for either single or multiple dose methotrexate. Main outcomes analysed were the success rates of single \& multiple dose MTX regimens. Secondarily the correlation between $\beta$ hCG levels on D1, D4, D7 and the success rates of medical management was analyzed.

Conclusion- Medical management of unruptured ectopic pregnancy with methotrexate has been successful in $80 \%$ of our study group, with least side effects and hence can be recommended as the first line of management in selected group of early unruptured ectopic pregnancies.
\end{abstract}

Key words:- ectopic pregnancy, medical management, methotrexate, outcomes.

\section{INTRODUCTION}

The practice of medicine is an art than a science because of its lack of constants. The inconstancy of symptoms, signs and opinions of reports is what makes ectopic pregnancy so fascinating to study and at times difficult to diagnose. Ectopic pregnancy (EP) is a classic gynecologic emergency and remains a major cause of mat ernal morbidity and mortality. 95$97 \%$ of ectopic pregnancies are tubal. During the past 2 decades, the clinical use of sensitive pregnancy testing, transvaginal sonography and diagnostic laparoscopy ha ve made a major impact on the diagnosis of ectopic pregnancy before tubal rupture. The combination of serum 
hCG titre and transvaginal sonography have supplanted physical examination findings as the most accurate diagnostic tool for determining the site of early gestation. Early diagnosis by TVS is potentially lifesaving, can reduce surgical interventions and allows for implementation of nonsurgical conservative treatment options. This has facilitated the move towards medical therapy with methotrexate for most women with unruptured ectopic pregnancies. The overall success rate of medical treatment in properly selected candidates is close to $90 \%$.Medical management apart from avoiding the inherent morbidity of anesthesia and surgery is also cost effective and offers success rate s comparable to surgical management, with no loss of future potential fertility.

\section{Aim of the study}

To analyse the outcomes of medically managed unruptured ectopic pregnancies treated with methotrexate and to correlate them with serum $\beta$-hCG levels.

\section{Materials and methods}

\section{Study design:}

Prospective observational study

Place of study:

Department of Obstetrics and Gynaecology of our institution

Duration of the study

October 2015-August 2017

\section{Sample size:}

\section{0}

\section{Inclusion criteria:}

- Confirmed tubal unruptured Ectopic pregnancy

- Hemodynamic stability

- Ultrasound Evidence of adnexal mass $<4 \mathrm{~cm} /$ minimal free fluid - Serum Beta HCG levels between $1500 \mathrm{mIU} / \mathrm{mL}$ and 10,000 $\mathrm{mIU} / \mathrm{mL}$.

- Commitment to follow up until ectopic pregnancy has resolved and consenting for medical management.

\section{Exclusion criteria:}

- All intrauterine pregnancies.

- Hemodynamic instability

- Fetal cardiac activity

- Beta-hCG level $<1500 \mathrm{mIU} / \mathrm{mL}$ and $>10,000 \mathrm{mIU} / \mathrm{mL} \bullet$ Methotrexate allergy

- Deranged renal and liver functions

- Heterotopic pregnancy

- Nontubal ectopic pregnancy

\section{Methodology:}

Patients suspected to have unruptured ectopic pregnancy were admitted and evaluated.

Detailed history and complete clinical examination were done. Transvaginal scan (TVS) of pelvis was done for all patients to know the size of the adnexal mass, presence or absence of 
cardiac activity, presence or absence of free fluid, to confirm absence of intrauterine gestational sac (IUGS) and the findings were recorded.

Patients who fulfilled the inclusion criteria for medical management of unruptured ectopic pregnancy $(\beta-\mathrm{hCG}>1500 \mathrm{mIU} / \mathrm{mL}$ and $<10000 \mathrm{mIU} / \mathrm{mL})$ with transvaginal scan features suggestive of unruptured ectopic pregnancy were included in the study for either single or multiple dose methotrexate (MTX) after obtaining approval from the Institutional Ethics Committee.

Those with $\beta$-hCG $<1500 \mathrm{mIU} / \mathrm{mL}$ were excluded as they could be in the discriminatory zone and could be having an evolving intrauterine pregnancy.

Informed consent was taken after explaining about the process of hospitalization, the need for administration of injection methotrexate, the need for constant monitoring and the need for surgery in case of rupture.

Apart from urine pregnancy test and serum beta human chorionic gonadotropin ( $\beta$-hCG) assay done on Day1, complete blood count (CBC), coagulation profile, and renal function tests (RFT), liver function tests (LFT) and blood grouping \& Rh typing were done.

Patients received intramuscular (IM) MTX at a dose of $1 \mathrm{mg} / \mathrm{KG}$ body weight.

Women received single dose or multiple doses of MTX after confirming the blood reports mentioned above.

Patients were kept in high dependency unit (HDU) and were monitored (pulse rate, blood pressure, abdominal circumference, pallor \& increasing abdominal pain). Adequate packed red blood cells (PRBC) were reserved. Serum $\beta$-hCG concentrations were measured on day four and day seven also.

Women who received multiple dose regimen were administered IM MTX of $1 \mathrm{mg} / \mathrm{kg}$ body weight on days 1,3,5 and 7.They received injection folinic acid IM on alternative days(days $2,4,6$ and 8 ) at the dosage of $0.1 \mathrm{mg} / \mathrm{kg}$ body weight as rescue and also to reduce the toxicity of MTX. Women who showed clinical signs and symptoms of tubal rupture were taken up for laparoscopy and then proceeded accordingly. Patients who showed decrease in serum beta hCG values on day 7 and who were clinically stable were discharged after educating about the symptoms \& signs of ruptured ectopic gestation (vaginal bleeding, abdominal pain, dizziness or syncope) and with the advice to report immediately if they occur.

Non sensitized Rhesus negative women received 100mcg anti-D immunoglobulin IM.

The toxicity of methotrexate treatment was evaluated by noting the side effects such as lower abdominal pain, vaginal bleeding, mouth ulcers, sore throat, gastrointestinal side effects or complaints of any rashes. The patients were followed up on weekly ba sis till $\beta$ - hCG levels reduced to $<2 \mathrm{mIU} / \mathrm{mL}$.

Main outcomes analysed were the success rates of single and multiple dose MTX regimens. Secondarily the correlation between $\beta$ hCG levels on D1, D4, D7 and the success rates of medical management was analysed.

\section{Statistical analysis:}

Statistical analysis was done using chi square test to determine the significant difference between the expected and the observed frequencies in categories. 


\section{Results}

Among the 50 study participants with ectopic pregnancies, $36(72 \%)$ were in the age group of 20-30 years. In this study, 26 cases were nulliparous (52\%) and 24 cases were multiparous (48\%). The maximum incidence was among the gravida II accounting for $20 \%$ (10 cases) followed by gravida III being $16 \%$ ( 8 cases). In our study, the predominant symptoms were amenorrhea and abdominal pain with incidence of $84 \%$ (42 cases) and 76\%(38 cases) respectively followed by bleeding and spotting per vaginum in 30\%(15 cases). Among the clinical signs,abdominal tenderness was present in 60\% (30 cases)and cervical motion tenderness was present in 54\%(27 cases). An adnexal mass was felt in 40\%(20 cases) on pelvic examination and fullness of fornices was observed in $52 \%$ (26 cases).

Table 1 Gestational Age at Presentation

\begin{tabular}{|l|l|l|}
\hline GESTATIONAL AGE (WEEKS) & NO.OF CASES (n=50) & PERCENTAGE \\
\hline 4 WEEKS + 5DAYS- 5 WEEKS & 3 & $6 \%$ \\
\hline 5 WEEKS + 1DAY - 6WEEKS & 16 & $32 \%$ \\
\hline 6WEEKS + 1DAY- 7 WEEKS & 13 & $26 \%$ \\
\hline 7 WEEKS + 1DAY -8WEEKS & 6 & $12 \%$ \\
\hline 8WEEKS + 1DAY - 9 WEEKS & 5 & $10 \%$ \\
\hline$>9$ WEEKS+1DAY & 7 & $14 \%$ \\
\hline
\end{tabular}

In our study, 29 cases presented between 5 weeks to 7 weeks of amenorrhea (58\%).(Table 1) In our study, 6 cases had history of Pelvic Inflammatory Disease (PID), 6 cases had history of previous ectopic gestation ,5 cases had history of Intra Uterine Contraceptive Device (IUCD) usage, 2 cases had history of tubal recanalization and 1 case had undergone tubal sterilisation. Among the 6 patients with previous ectopic gestation, one had received medical management, 4 had undergone salpingectomy of the affected tubes and one patient with history of tubal recanalization had undergone medical management previously.

In our study, 42 cases conceived spontaneously (84\%),6 cases conceived by ovulation induction (12\%) and 1 case each conceived by ovulation induction with IUI (2\%) and in vitro fertilisation (2\%). In our stud y, transvaginal ultrasound was done in all patients of whom 38 cases had adnexal mass (76\%), 8 cases had adnexal gestational sac within the mass (16\%) and 4 cases presented with adnexal mass with free fluid (8\%). In our study, 31 cases presented with mass size of 1 to $2 \mathrm{~cm}$ (62\%),12 cases presented with mass size of 2.1 to $3 \mathrm{~cm} \mathrm{(24 \% )} \mathrm{and}$ 7 cases presented with mass size of 3.1 to $4 \mathrm{~cm}(14 \%)$. 
Table 2 Distribution of Cases According To Regimen

\begin{tabular}{|l|l|l|}
\hline REGIMEN & $\begin{array}{l}\text { NO.OF } \\
\text { CASES(n=50) }\end{array}$ & PERCENTAGE \\
\hline SINGLE DOSE MTX & 15 & $30 \%$ \\
\hline $\begin{array}{l}\text { MULTIPLE DOSE MTX + FOLINIC } \\
\text { ACID RESCUE }\end{array}$ & 35 & $70 \%$ \\
\hline
\end{tabular}

In our study, among the 50 cases, 15 cases (30\%) received single dose regimen and 35 cases $(70 \%)$ received multiple dose regimen.(Table 2). The overall success rate for medically managed unruptured ectopic pregnancy was $80 \%$ (40 cases) and failure rate was $20 \%$ (10cases). Out of the above 40 cases, 13 cases (32.5\%) were successfully managed with single dose of MTX regimen and 27 cases (67.5\%) were successfully managed with multiple doses of MTX with folinic acid rescue regimen. Our study did not show any correlation between gestational age and success of medical management ( $p$ value 0.429 ). However larger studies need to be done to substantiate this. No statistically significant difference was observed between the success and failure groups in our study according to the size of the mass ( $\mathrm{p}$ value 0.257 ).

Figure 1

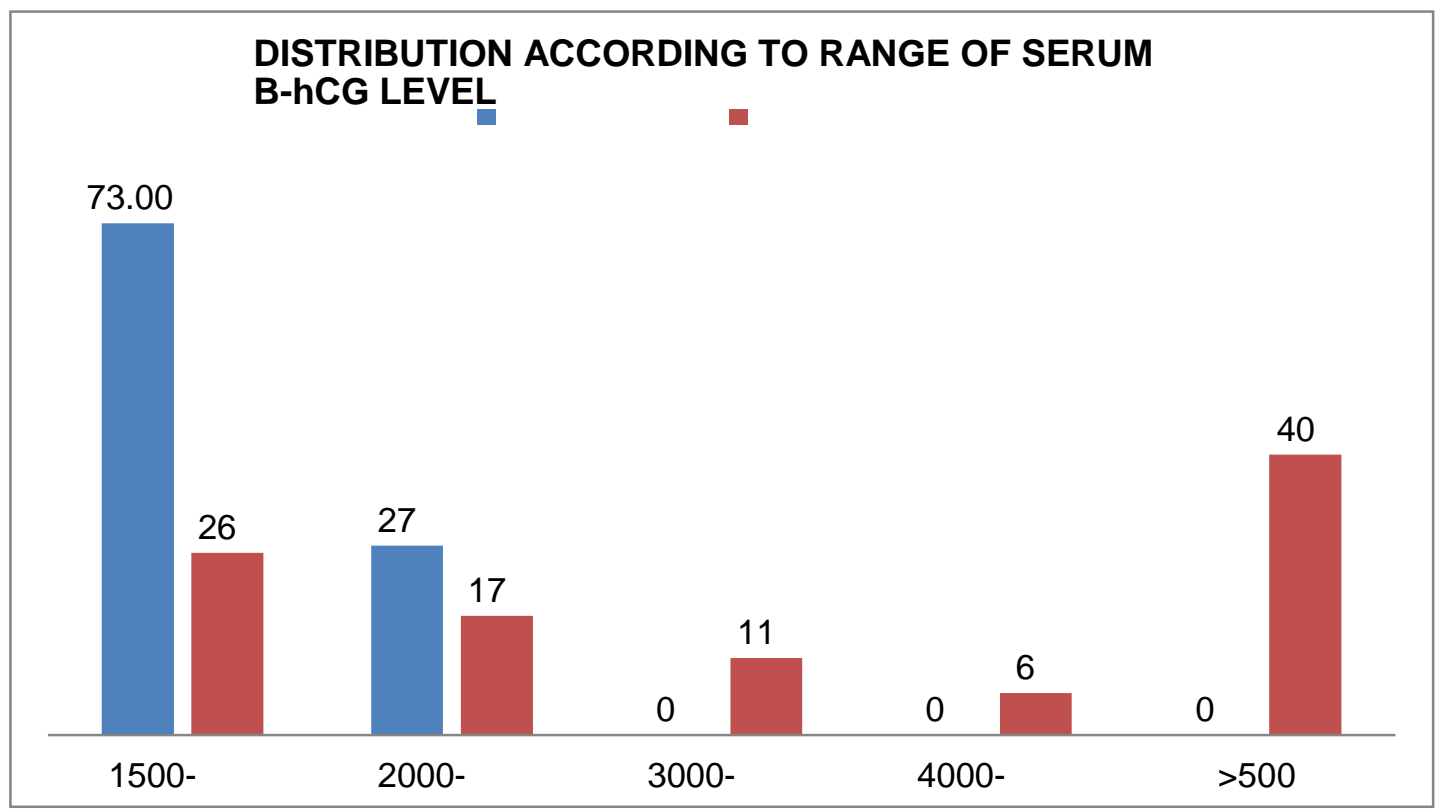

All the 15 women treated by single dose MTX had their initial $\beta$-hCG levels $<3000$ $\mathrm{mIU} / \mathrm{mL}$.Among the 35 women treated with multiple dose MTX/FA regimen, 14 women $(40 \%)$ had their initial $\beta$-hCG levels $>5000 \mathrm{mIU} / \mathrm{Ml}$ and 21 women $(60 \%)$ had their initial $\beta$-hCG levels $<5000 \mathrm{mIU} / \mathrm{mL}($ Figure 1). 
Table 3 Comparison of Single Dose Mtx And Multiple Dose Mtx Outcomes

\begin{tabular}{|l|l|l|l|}
\hline REGIMEN & $\begin{array}{l}\text { SUCCESS } \\
\text { GROUP }\end{array}$ & FAILURE GROUP & $\begin{array}{l}\text { P } \\
\text { VALUE }\end{array}$ \\
\cline { 1 - 3 } SINGLE DOSE MTX & $13(86.66 \%)$ & $2(13.34 \%)$ & 0.310 \\
\cline { 1 - 3 } MULTIPLE DOSE MTX & $27(77.14 \%)$ & $8(22.86 \%)$ & \\
\hline
\end{tabular}

Single dose regimen was successful in $86.66 \%$ cases and multiple dose regimen was successful in $77.14 \%$ cases with a p value of 0.310 showing no statistical significance (Table 3 ).

Table 4 Mean Value of Serum Beta Hcg (On D1, D4, And D7)

\begin{tabular}{|l|l|l|l|}
\hline $\begin{array}{l}\text { MEAN SERUM } \\
\boldsymbol{\beta} \text {-hCG VALUES } \\
\mathbf{m I U} / \mathbf{m L}\end{array}$ & $\begin{array}{l}\text { SINGLE DOSE MTX } \\
\mathbf{m I U / m L ( n = 1 5 )} \\
\text { Initial B-hCG levels }\end{array}$ & $\begin{array}{l}\text { MULTIPLE } \\
\text { DOSE MTX } \\
\mathbf{m I U} / \mathbf{m L}(\mathbf{n}=\mathbf{3 5}) \\
2924)\end{array}$ & P VALUE 0.001 \\
\hline B-hCG levels on D4 & $1433(4511-$ & \\
\hline B-hCG levels on D7 & $943(360-2851)$ & $4785(593-10131)$ & 0.001 \\
\hline
\end{tabular}

Table 4 shows the mean value of serum $\beta$ - hCG level on day 1 , day 4 and day 7 among the 2 groups and the $p$ value is significant $(0.001)$.

Table 5: Comparison of Mean Serum B-Hcg Values between Success and Failure Groups:

\begin{tabular}{|l|l|l|l|}
\hline $\begin{array}{l}\text { MEAN SERUM } \\
\boldsymbol{\beta} \text {-hCG VALUES } \\
\mathbf{m I U} / \mathbf{m L}\end{array}$ & $\begin{array}{l}\text { SUCCESS } \\
\text { GROUP } \\
\mathbf{m I U / m L}(\mathbf{n}=\mathbf{4 0})\end{array}$ & $\begin{array}{l}\text { FAILURE } \\
\text { GROUP } \\
\mathbf{m I U / m L ( n = 1 0 )}\end{array}$ & P- VALUE \\
\hline Initial B-hCG levels & $3705(1511-9825)$ & $3559(1517-8015)$ & 0.923 \\
\hline B-hCG levels on Day4 after MTX & $3385(491-9421)$ & $6080(1168-10131)$ & 0.060 \\
\hline B-hCG levels on Day 7 after MTX & $3018(198-10048)$ & $7281(2640-10943)$ & 0.017 \\
\hline
\end{tabular}


Statistically significant difference was noted for D7 $\beta$-hCG between success $\&$ failure groups $(\mathrm{p}$-value $=0.017) .($ Table 5$)$.

No statistically significant difference in the beta -hCG was observed between the success and the failure groups when comparing D1 to D4 beta hCG values (p value 0.57). Among the 40 women who had success with medical management, only 22 cases $(55 \%)$ had $>15 \%$ fall in beta hCG values between day D1 and D4.The remaining 18 cases (45\%) who had rising trend in beta hCG values between D1 and D4 had successful outcome with MTX.

No statistically significant difference in the beta -hCG was observed between the success and the failure groups when comparing D4 to D7 beta - hCG values(p value 0.457).Out of the 40 women who had successful medical management with MTX, 50\% (20 cases) showed increasing trend of $\beta$ - hCG from D4 to D7.

No statistically significant difference in the beta-hCG was observed between the success and the failure groups when comparing D1 to D7 beta - hCG values ( $\mathrm{p}$ value 0.334).Among the 40 cases who had successful medical management with MTX, 57\% (23 cases) of women showed falling trend of $\beta$ - hCG (>15\%) from D1 to D7.The remaining 43\%(17 cases) who successfully medically had rising trend of beta hCG from D1 to D7.

\section{Table 6 Distribution Of Cases According To Initial Serum B Hcg Level In The Success Group}

\begin{tabular}{|l|l|l|}
\hline $\begin{array}{l}\text { SERUM } \beta \text { hCG } \\
\text { LEVELS } \\
(\mathbf{m I U} / \mathbf{m L})\end{array}$ & $\begin{array}{l}\text { SINGLE DOSE MTX } \\
(\mathbf{n}=\mathbf{1 3})\end{array}$ & MULTIPLE DOSE MTX (n=27) \\
\hline $1500-1999$ & 10 & 7 \\
\hline $2000-2999$ & 3 & 4 \\
\hline $3000-3999$ & 0 & 2 \\
\hline $4000-4999$ & 0 & 2 \\
\hline$>5000$ & 0 & 12 \\
\hline
\end{tabular}

12 women were treated successfully by multiple dose regimen though initial $\beta$-hCG levels were $>5000 \mathrm{mIU} / \mathrm{mL}$. 13 women were successfully treated by single dose and their initial $\beta$ hCG was $<3000 \mathrm{mIU} / \mathrm{mL}$. (Table 6).

Among the 10 patients who had failed medical management, 6 women $(60 \%)$ had features of ruptured ectopic gestation with increasing abdominal pain, during the monitoring of MTX treatment and hence were taken up for laparoscopy. 3 had tubal rupture and 3 had tubal abortion. 4 cases $(40 \%)$ of them were taken up for laparoscopy for fear of tubal rupture in view of increasing $\beta$-hCG levels.9 patients (90\%) had laparoscopic partial salpingectomy and one patient (10\%) had laparoscopic salpingotomy.

In our study, more frequently encountered side effects of MTX were nausea (26\%), vomiting (26\%), gastritis (20\%), abdominal pain (14\%), headache (8\%), diarrhoea( $4 \%$ ) and 
stomatitis(2\%).The most common complaint was pelvic pain and cramps which was caused by the changes occurring in the ectopic gestation due to th e treatment rather than by MTX itself. None of the cases had hepatic or renal dysfunction or bone marrow suppression.

In our study, among the 50 cases, 27 cases stayed in the hospital for 8 to 10 days (54\%).10 cases stayed in the hospital for more than 10 days (20\%) and 13 cases stayed between 5 to 7 days $(26 \%) .4$ cases $(8 \%)$ received blood transfusion and 46 cases $(92 \%)$ were managed without blood transfusion.

\section{Table 7 Number of Days Required For Normalization of Beta Hcg}

\begin{tabular}{|l|l|l|}
\hline NO OF DAYS & SINGLE $(\mathbf{n = 1 3})$ & MULTIPLE $(\mathbf{n}=\mathbf{2 7})$ \\
\hline $21-28$ days & 10 & 5 \\
\hline $29-35$ days & 3 & 9 \\
\hline $36-42$ days & 0 & 9 \\
\hline $43-49$ days & 0 & 4 \\
\hline
\end{tabular}

In our study, 13 cases were successfully managed with single dose MTX and all of them (100\%) required $<35$ days for normalization of $\beta$ - hCG levels. Among the 27 cases managed successfully with multiple dose MTX regimen,14 cases (52\%) required <35 days for normalization of $\beta$ - hCG levels and 13 cases (48\%) required 35 days- 49 days for normalization of $\beta$-hCG levels.

\section{Discussion}

Out of the 50 cases included in our study, maximum incidence (72\%) was in the age group of 20-30 years. This is comparable with studies conducted by Shraddha Shetty K et al (74.2\%)1, Khaleeque et al,2 (74.2\%) and Manohar et al3 (64.51\%) with similar incidence in the age group of 20-30years.In our study, study maternal age did not affect the outcome of methotrexate treatment.This was similar to the study by Hansa Dhar etal 4 .In our study, no correlation was noted between gestational age and the outcome of medical management. The studies by Pooja et al5 and Hansa Dhar et al4, also had similar findings.

In our study, no statistically significant difference was noted between the success and failure groups according to the size of the adnexal mass ( $\mathrm{p}$ value $0 . .257$ ). In the study by Pooja et al 5, success rate of $96.6 \%$ with medical management was found when the adnexal mass size was less than $3 \mathrm{~cm}$. In the study by $\mathrm{H}$. Fletcher et al 6 , the presence of an adnexal mass of size greater than $5 \mathrm{~cm}$ was associated with an increased risk of treatment failure. In the study by Hansa et al4, in women with $0-4 \mathrm{~cm}$ adnexal mass success rate was $71.4 \%$ and in patients with adnexal mass more than $4 \mathrm{~cm}$, the success rate was less $(\mathrm{p}=0.132)$. 
According to the study by Vaswani et al7 an overall success rate of 75\% (30/40) was reported for MTX therapy for ectopic gestation. .Success rate of MTX therapy in our study was $80 \%$ which is comparable with other studies.

In our study, a success rate of $86.6 \%$ (13/15 cases) was noted with single dose MTX with the mean initial beta hCG of $1882 \mathrm{mIU} / \mathrm{ml}$ and multiple dose MTX regimen had a success rate of $77.1 \%$ (27/35 cases) with the mean initial beta-hCG of $4445 \mathrm{mIU} / \mathrm{mL}$ with a significant $\mathrm{p}$ value of 0.001 . Study by Sumerya et al8 was similar showing significance whereas studies by Junhwan etal9, Dogan etal10 and Hansa etal4 did not find this as significant.

In our study, the difference between the mean pre-treatment serum beta hCG level in the success and failure groups was not statistically significant (3705 versus $3559 \mathrm{mIU} / \mathrm{mL}$ with $\mathrm{p}$ value 0.923 ). The study by Sumerya et al 8 was also similar. The studies by Pooja et al5 and Dogan etal10 had significant difference in the initial mean serum beta hCG between those who had successful and failed medical management. This disparity could be due to more number of patients in other studies and also because the inclusion criteria for initial bet a hCG was $<1500$ $\mathrm{mIU} / \mathrm{mL}$ in other studies. According to a study conducted by Lipscomb et al11, initial level of beta hCG was the best prognostic factor in predicting the success ( $94 \%$ success rate if below $10,000 \mathrm{mIU} / \mathrm{ml}$ versus $75 \%$ success rate if above $10,000 \mathrm{mIU} / \mathrm{ml})$. They concluded that an initial beta hCG level above 10, 000mIU/ml was a risk factor for MTX treatment failure. $82 \%$ women who were successfully treated required only one dose in their study.

In our study, the difference of mean serum beta hCG level on day 4 between success and failure groups (3385 mIU/mL versus $6080 \mathrm{mIU} / \mathrm{mL}, \mathrm{p}$ value 0.060), was not statistically significant.This was similar to the findings of Sumerya etal8 whereas this was statistically significant as per the studies of Pooja etal5 and Dogan etal10. In Our study, the difference of mean serum $\beta$ - hCG on D7 between success and failure groups $(3018 \mathrm{mIU} / \mathrm{mL}$ versus 7281 $\mathrm{mIU} / \mathrm{mL}, \mathrm{p}$ value 0.017 ) was statistically significant.

This was similar to the findings of Sumerya et a18, Pooja etal5 and Dogan etal10.

In our study 22 out of the 40(55\%) successful cases had $>15 \%$ fall in $\beta$-hCG between days 1 and 4 after MTX administration. whereas 18 out of the 40(45\%) successful cases actually had rising trend of beta hCG between D1 and D4. According to a study by Monika Skubisz et al12 a decline in serum beta hCG between days 1-4 after MTX injection provides an early indication of likelihood of treatment success.

Out of the total 50 cases in our study, 24 cases had rising trend of serum beta hCG $(>15 \%$ increase on day 4) among which 18 cases were successfully managed medically and 6 cases underwent surgery. It is not unusual to find an increase of beta hCG on D4 but not all of them would require surgery. In the study by Sumanth et al13 10.4\% of treatment failure was related to a rise in serum $\beta$-hCG values between days 1 and 4.In Pooja et al5, 31.25\% of treatment failure was related to a rise in serum beta hCG values between days 1 to day 4 .

Also among the 40 successful cases in our study 20 cases(50\%) only had falling trend of beta hCG between D4 and D7 and the remaining 20 cases(50\%) had rising trend of beta hCG between D4 and D7. As per Hansa et al4, treatment failure based strictly on a high increase in $\beta$-hcg level from day 4 to 7 may be a hasty judgment.

In our study, out of the 10 cases with unsuccessful medical management, 3 had tubal rupture and 3 had tubal abortion with hemodynamic instability and 4 had rising trend of $\beta$ - hCG values. Among the 10 cases, 9 cases(90\%) underwent laparoscopic partial salpingectomy and 1 case 
(10\%) had laparoscopic salpingostomy. According to Manohar et al3, salpingectomy was performed in $48.38 \%$ cases.

According to Col pankaj et al14, 75\% of study group (24/32) got successfully treated with methotrexate. Among the remaining 25\%(8/32) 4 had rapidly rising $\beta$-hCG values , 2 had persistent abdominal pain and 2 had suspected rupture with features of shock .

Pain after MTX treatment could be due to tubal abortion or stretching of the tube by hematoma contributing to increased failure rate in most of these studies. Fear of rupture misleads clinicians to operate early on unruptured ectopic pregnancies that would otherwise resolve with medical management. Differentiating 'separation pain' due to tubal abortion from pain due to tubal rupture can be difficult and may lead to early surgical intervention.

The side effects of MTX are related to the dose and mode of administration. With single -dose MTX, most authors have reported no significant adverse effects. In our study we did not encounter abnormal renal or liver function tests and bone marrow depression, hence all patients completed the study. As per our study $80 \%$ patients had a hospital stay less than 10 days. According to Tahmina et $15,94.4 \%$ of their patients had a hospital stay of less than 8 days, while $5.6 \%$ of the women needed prolonged hospitalization up to 14 days

No maternal mortality was noted in our study, consistent with A. Abbas and H. Akram study16. Maternal mortality due to EP was reported to be between $0 \%$ and $1.3 \%$ in various studies. Mortality is mostly due to haemorrhage following rupture of the EP due to delayed diagnosis and referrals.

\section{Conclusions}

1. Medical management of unruptured ectopic pregnancy with methotrexate has been successful in $80 \%$ of our study patients, with least side effects and hence can be recommended as the first line of management in selected group of early unruptured ectopic pregnancy.

2. The mean beta hCG values on Day4 and Day7 of treatment were significantly higher in the failure group compared to the success group.

3. It is not unusual to find an increase of beta hCG on day 4 and day 7 of treatment but not all of them would require surgery, they would require vigilant monitoring for signs of tubal rupture.

\section{References}

1) Shraddha Sh etty K et.al/ Clinical Study of Ectopic Pregnancies in a Tertiary care hospital of Mang alore. Innovative Journal of Medical and Health Scien ce 4: 1 Jan - Feb (20 14) 305 309.

2) Khaleequ e F, Siddiqui RI, Jafarey SN. Ectopic pregn ancies: A th ree year study.J-Pak Med Assoc . 2 001; 51(7):240-42.

3) Manohar R, Kavyashree G, Lalith a Shivann a. "Retrospectiv e Study of Ectopic Pregnan cy in a Teaching Hospital". Journ al of Evolution of Medical and Dental Scien ces 2014; Vol. 3, Issue 63, November 20; Page: 13817 -13822, DOI: 10.14260/jemd s/2014/3848 
4) Hansa Dhar, Ilh am Hamdi, Bhawn a Rathi Methotrexate Treatment of Ectopic Pregnan cy: Experien ce at Nizwa Hospital with Literature Review Oman Medical Journal (2011) Vo 1. 26, No. 2: 94 -98.

5) Pooja Rajesh Vaswani, Predictors of Success of Medical Man agement of Ectopic Pregn ancy. Journal of Clinical and Diagnostic Research. 2014 Aug, Vol-8(8).

6) Fletcher, K. Buch anan, L. Jacob, Conserv ative treatment of unruptured ectopic pregnan cy in Jamaica, Op en Journal of Ob stetrics and Gynaecology, 2011, 1, 6-11 doi:10.4236/ojog.2011.11002 Published Online March 2011 (http://www.SciRP.org/journal/ojog/.

7) Vaswani PR. Predictors of success of medical manag ement of ectopic pregnancy in a tertiary care hospital in United Arab Emirates. J Clin Diagn Res 2014; 8: OC04 -OC08.

8) Sümeyra Nergiz AvcioLlu, 1 Sündüz Özlem Altinkaya,1 Mert Küçük,2Selda Demircan Sezer,1 and Hasan Yüksel1 - Predictors of Success of Differen t Treatment Mod alities for Manag ement of Ectopic Pregnancy. Hindawi Publishing Corporation Ob stetrics and Gynaecology International Volume 2014, Article ID 423708, 6 pages http://dx.doi.org/10.1155/2014/423708

9) Junhwan Kim1, Young Mi Jung1, Da Yong Lee2, Byu ng Chul Jee2. Pre - treatment serum hu man ch orionic gonadotropin cut-o ff valu e for medical treatment success with sin gle -dose and multi-dose regimen of methotrexate in tubal ectopic pregn ancy. Original Article Obstet Gy necol Sci 2017; 60(1):79-86 https://doi.org/10.5468/ogs.2017.60.1.79 pISSN 2287-8572 . eISSN 2287 - 8580.

10) Askin Dog an a, *, Ibrahim Gu lhan a, Ib rahim Uy ar a, Atalay Ekin a, Cenk Gezer a, Muzaffer Bilgin b, Cu"neyt E. Tan er a, Ibrahim E. Ertas, Methotrexate treatment in progressive tubal ectopic pregnan cies and hCG - related clinicosu rgical implications, $1607-551 \mathrm{X} / \mathrm{Co}$ pyright ${ }^{\text {a }}$ 2016, Kaohsiung Medical University. Published by Elsevier Taiwan LLC http://dx.doi.org/10.1016/j. kjms. 2016.05.004

11) Lipsco mb GH, McCord ML, Stovall TG, et al. Predictors of su ccess of methotrex ate treatment in women with tubal ectopic pregnan cies. N Engl J Med. 1999; 341: 1974 -78.

12) Skubisz MM, Li J, Wallace EM, Tong S: Declin e in Beta hCG lev els between days 0 and 4 after a single dose of methotrex ate for ectopic pregnan cy predicts treatment success: a retro spectiv e cohort study. BJOG. 2011; 118(13):1665 -68.

13) Medical Manag ement of Ectopic Pregn ancy with Methotrexate Su mant R Shah*, Sandip Sonara†, Bhavesh Patel†, Nidhi Patel†. Indian Journ al of Clinical Practice, Vol. 24, No. 11, April 2014.

14) Systemic methotrexate: An effective altern ative to surgery for manag ement of unruptured ectopic pregn ancy Col Pankaj Talwar, VSM a, ${ }^{*}$, Lt Col K. Sandeep b, Lt Col Nikita Naredi c, Col B.S. Duggal (Retd), Lt Col Tony Jose e http://dx.doi.org/10.1016/j.mjafi.2012.08. 032.

15) ectopic pregnan cies and hCG-related clinico surgical implications, 1607 - 551X/Copyright ${ }^{\text {a }}$ 2016, Kaohsiung Medical Univ ersity. Published by Elsevier Taiwan LLC http://dx.doi.org/10.1016/j. kjms. 2016.05.004

16) S Tahmina et al., A six -year Retro spectiv e Study of Ectopic Pregn ancies. Journal of Clinical and Diagnostic Research. 2016 Oct, Vol -10(10): QC13 - QC16

17) Abbas A,Ak ram H.Ectopic pregnan cy; audit at Maula Bakhsh Teaching Ho sp . Sargodha. Pro fession al Med J Mar 2011;18(1):24 -27 